\title{
Glossopharyngeal Neuralgia Associated with Cardiac Syncope
}

\author{
Jorge Elias, Ricardo Kuniyoshi, Wilson Valadão Hermes Carloni, Mauricio Rocha Borges, \\ Carlos Alberto Peixoto, Derval Pimentel
}

Vitória, ES - Brazil

Glossopharyngeal neuralgia is an uncommon condition that has rarely been described in association with syncope. We report here 2 cases of glossopharyngeal neuralgia in elderly patients. Both were male and underwent temporary pacemaker insertion to prevent syncopal episodes. We discuss the clinical and surgical treatment of glossopharyngeal neuralgia, the role of cardiac stimulation, and the possible physiopathological mechanism of the associated cardiac disturbances.

Glossopharyngeal neuralgia is an uncommon condition first described by Weisenberg in 1910. It is characterized by paroxysms of lancinating pain localized unilaterally in the base of the tongue, soft palate, throat, and in the lateral and posterior regions of the pharynx, radiating to the ipsilateral ear. The pain can be triggered by swallowing, coughing, yawning and chewing and, usually lasts seconds or minutes. Association between glossopharyngeal neuralgia and syncope is very rare and is due to short episodes of bradycardia, asystole, and hypotension ${ }^{1-3}$. We report here 2 patients with glossopharyngeal neuralgia associated with cardiac syncope treated with the insertion of a temporary pacemaker associated with specific therapy. We discuss the pathogenesis of syncope associated with glossopharyngeal neuralgia and its therapeutical possibilities.

\section{Case Reports}

Case 1 - A 68 -year-old white male was admitted to the hospital in February 1995 with a 2 - week history of frequent episodes of severe pain in the mouth and face, associated with recurrent syncopal attacks during the previous 3 days.

Instituto de Cardiologia do Espírito Santo - Clínica do Ritmo - Vitória Mailing address: Jorge Elias - Rua Alfen Alves Pereira, 60 - Enseada do Suá CEP: 29050-190 Vitória, ES - E-mail: eliasj@zaz.com.br
He complained of paroxysms of severe pain in the mouth radiating down to the left side of the neck and ear, sometimes followed by dizziness and episodes of loss of consciousness for a few seconds. These events occurred several times a day, with progressive intensity and frequency, triggered by the swallowing of cold liquids and by talking. He also reported that, sometimes, during the episode of pain he used to compress the lateral region of his neck just before the syncopal episode. He had a past history of hypertension and used an ACE inhibitor.

Neurological and nasopharyngeal assessment through a clinical examination, indirect laryngoscopy, computed tomographic scan of the brain, and magnetic resonance scan of the brain revealed nothing abnormal.

The physical examination showed regular cardiac rhythm, with a heart rate $65 \mathrm{bpm}$ and blood pressure of $160 /$ $80 \mathrm{mmHg}$. During clinical evaluation, he experienced an episode of pain that evolved to syncope associated with seizure. Massage of the carotid sinus performed after the syncopal event in upright and orthostatic positions was normal. He was placed on Holter monitoring, which recorded 5 episodes of sinus arrest and atrioventricular block associated with syncopal episodes (fig. 1).

After glossopharyngeal neuralgia associated with cardiac syncope was diagnosed, insertion of a temporary ventricular bipolar electrode was performed through the right jugular vein, enabling total and immediate control of syncopal events. Therapy with carbamazepine was initiated, at an initial dose of $400 \mathrm{mg} /$ day, reaching $800 \mathrm{mg} /$ day, with progressive improvement in the clinical picture of pain. Broad clinic-laboratory assessment did not reveal the etiology of the glossopharyngeal neuralgia.

After 4 days of treatment with carbamazepine, the patient experienced relief of the pain, and the pacemaker electrode was withdrawn.

He was discharged from the hospital on a maintenance dose of carbamazepine at $800 \mathrm{mg} /$ day. 


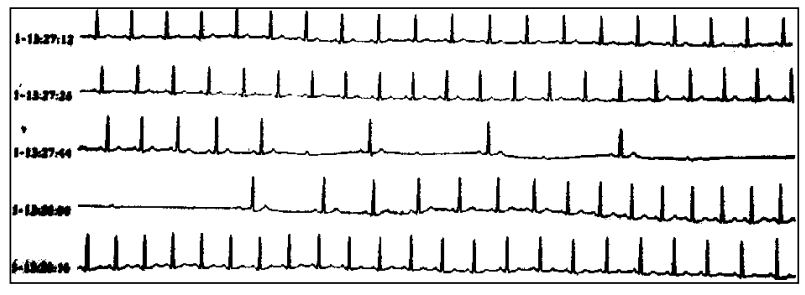

Fig. 1 - Record of electrocardiogram showing an episode of bradyarrhythmia associated with glossopharyngeal neuralgia crises in patient 1 . Observe the association between sinus bradycardia and second grade atrioventricular block, type $2: 1$ and 3:1 temporary.

After 2 weeks of treatment, the patient stopped using carbamazepine and experienced an immediate recurrence of pain associated with episodes of syncope and seizures. Clinical control was obtained after establishment of pharmacological treatment.

The patient was followed up on a regimen of carbamazepine and has been asymptomatic for 5 years, even though carbamazepine was discontinued 2 years ago.

Case 2 - An 80-year-old white male was admitted to the hospital in September 1999 after episodes of lancinating pain in the pharynx followed by syncopal episodes and cranial trauma and a right maxillary sinus fracture.

He reported that the pain had started 3 years earlier in the pharynx and was related to swallowing but responded well to carbamazepine.

After carbamazepine was discontinued, due to the development of bone marrow aplasia, the patient experienced recurrence of pain, a week later, associated with nausea and vomiting.

Carotid Doppler, a computed tomographic scan of the brain, and nasopharyngeal examination showed no pathological evidence. Massage of the carotid sinus did not cause bradycardia, and the ECG showed no abnormalities.

Three days after admittance, the episodes occurred daily, were uncontrollable, even after the introduction of diphenylhydantoin.

Oral cavity manipulation or swallowing of a cold liquid caused episodes of typical pain, radiating down to the left ear, with a temporary reliefafter a lingual nerve block with Marcaine.

The quantity and severity of pain episodes became progressively worse with several episodes on the same day followed by a seizure leading to the need for mechanical ventilatory support. Due to severe sinus bradycardia and asystole, during the pain episode, atropine and isoproterenol were used unsuccessfully (fig. 2). After the insertion of a temporary pacemaker through dissection of the right basilar vein, bradyarrhythmia and seizures were controlled (fig. 3 and 4). He was treated by alcohol injection through the jugular foramen, due to the severity of his clinical condition and because anesthetic block, at this level, caused significant improvement in the symptoms.

After the procedure, the patient experienced remission

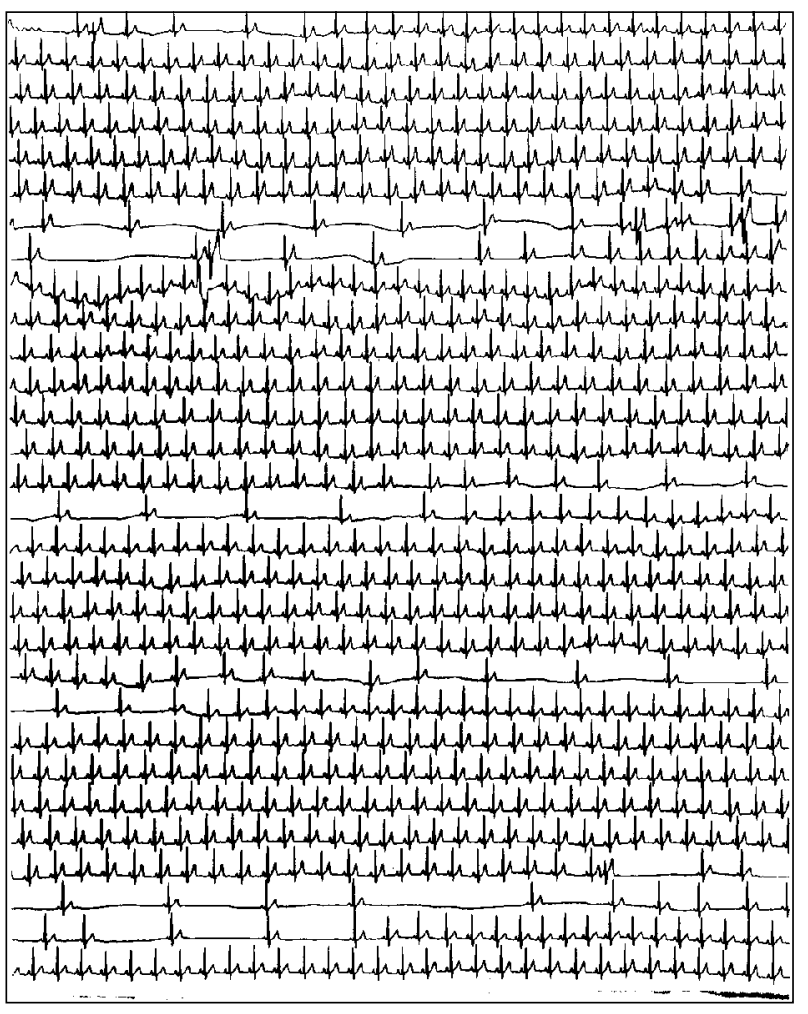

Fig. 2 - Continuous record showing frequent episodes of severe sinus bradycardia and sinus pause associated with glossopharyngeal neuralgia and seizure in patient 2 .

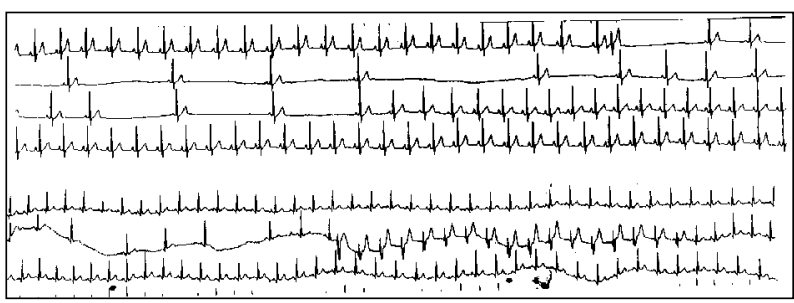

Fig. 3 - A) Detail of the episode of severe bradycardia in patient 2; B) beginning of temporary ventricular stimulation during crises showing stabilization of heart rate in patient 2 .

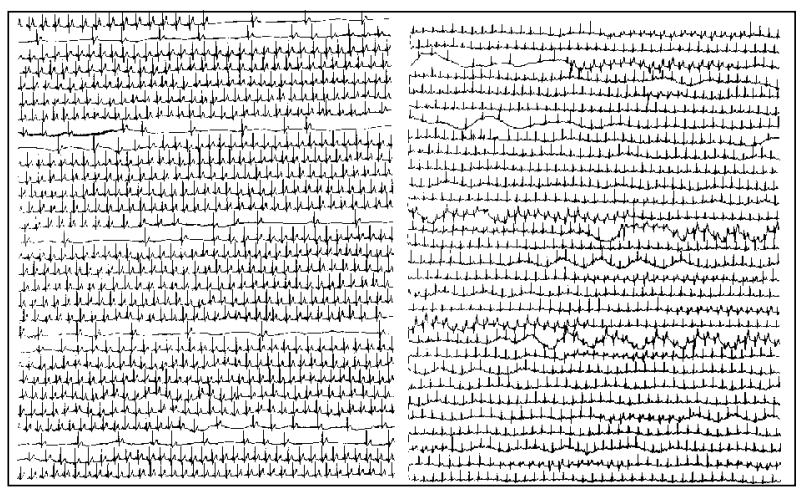

Fig. 4 - Continuous record showing trace before (A) and immediately after (B) introduction of temporary pacemaker showing control of bradyarrhythmia.

of the events, enabling the withdrawal of the temporary pacemaker electrode and extubation 3 days after the intervention. 
He evolved with transitory difficulty in of swallowing with later normalization. During follow-up, the patient remained free of glossopharyngeal neuralgia symptoms.

\section{Discussion}

Glossopharyngeal neuralgia is a rare form of pain when compared to trigeminal neuralgia $(0.2-1.3 \%$ of cases of facial pain) ${ }^{4}$. The correlation between these 2 types of neuralgia ranges from $1: 70$ and $1: 100^{1,4}$.

Glossopharyngeal neuralgia was first described by Weisenberg, in 1910, in a patient with a cerebellopontine angle tumor ${ }^{1}$. Although in the majority of cases of glossopharyngeal neuralgia occur in isolation (idiopathic), they may be secondary to cerebellopontine angle tumors, intracranial vascular compression, carcinoma of the laryngeal and nasopharyngeal tumors that spread locally, parapharyngeal abscess, trauma, multiple sclerosis, Paget's disease or cranial base tumors, calcified stylohyoid ligament, direct carotid puncture, and dental extractions ${ }^{1-3}$. It is a disease that occurs only in adults, especially in patients over $50^{1}$. Some authors report that the disorder is more common in males, but others did not find a significant statistical difference between the sexes ${ }^{1,3}$. The disease can have a sudden onset. Glossopharyngeal neuralgia and trigeminal neuralgia are usually similar to trigeminal neuralgia and are characterized by paroxysms of unilateral pain following the glossopharyngeal nerve course and affecting the left side more frequently than the right.

Patients with glossopharyngeal neuralgia have more difficulty in identifying the triggering mechanisms than patients with trigeminal neuralgia, probably because they are less able to accurately locate the pain in deep structures of the mouth, pharynx, and ear. The paroxysms of pain are usually precipitated by swallowing, especially cold drinks, chewing, coughing, and speaking. Pain is described as severe, sharp as like a "stab" or a "needle", lasting seconds to minutes. In cases of doubtful diagnoses, pharynx stimulation, triggering typical symptoms, can be highly suggestive of this condition. Massage of the carotid sinus is considered inefficient in glossopharyngeal neuralgia, which enables its distinction from hypersensitive carotid sinus syndrome. However, Jamshidi and Masrror ${ }^{5}$ described 2 elderly women with glossopharyngeal neuralgia and asystole associated with the presence of hypersensitive carotid sinus ${ }^{6}$.

It is common that patients with glossopharyngeal neuralgia experience pain remission for a period that ranges from months to years.

Association between glossopharyngeal neuralgia and syncope is even rarer. Harris, in 1921, was the first author to report a case of idiopathic neuralgia associated with syncope, describing the pathological mechanism, and Riley in 1942 presented 2 cases in which glossopharyngeal neu- ralgia was associated with syncope due to cardiac arrest and seizure ${ }^{1,3,7}$. Rushton ${ }^{1}$, in 1981, reported on 217 patients with glossopharyngeal neuralgia admitted to the Mayo Clinic, of whom only 4 had associated syncope.

Syncope is due to extreme bradycardia or asystole, as in the present report; however, in a few cases, syncope can be caused by severe hypotension ${ }^{8}$.

Usually, a latency period occurs between the beginning of the symptoms of glossopharyngeal neuralgia and the first syncopal event, which may vary from a few weeks to several years ${ }^{9}$, which was observed in our patients.

Syncopal events are preceded by an intermittent, progressive, and lancinating pain located in the retromandibular or oropharyngeal region, and, sometimes, radiating to the ipsilateral ear and tempora-occipital region. Some patients experience ipsilateral lacrimation and hyperemia.

The acute phase is generally brief, lasting seconds to minutes. The events may occur in isolation or recur variably. The patient is increasingly disturbed by pain, rapidly followed by syncopal episodes. The majority of cases experience pallor, followed by hypotension associated with bradycardia, leading to loss of consciousness associated sometimes with clonic movements. Electroencephalogram recorded during the attacks revealed a brain hypoxia pattern ${ }^{1}$.

The most acceptable mechanism underlying the association between bradyarrhythmias, hypotension and syncope, and glossopharyngeal neuralgia is the close connection between the vagus nerve and glossopharyngeal nerve, particularly with respect to visceral sensations, which may favor the creation of a vagoglossopharyngeal reflex $\mathrm{arch}^{3}$.

Afferent nerve impulses from irritative or ischemic lesions arising from the peripheral course of the glossopharyngeal nerve may reach the nucleus of the tractus solitarius of the midbrain through this nerve, and, via collaterals, reach the dorsal nucleus of the vagus nerve. This is the main autonomic nucleus, supplying parasympathetic fibers to the heart, bronchi, and abdominal nerve. Furthermore, the carotid sinus nerve (Hering's nerve), responsible for the conduction of impulses from the body and the carotid sinus, runs in the main trunk of the glossopharyngeal nerve and ends in the dorsal nucleus of the vagus nerve ${ }^{3,6}$. It has been suggested that artificial synapses develop in the proximal portion of the glossopharyngeal nerve stimulating the carotid sinus via somatosensorial impulses of glossopharyngeal neuralgia. Thus, the presence of an irritative phenomenon might induce bradycardia with possible mediation of the vagus nerve.

The afferent glossopharyngeal stimulus may inhibit vasomotor centers, which leads to peripheral vasodilatation and a pronounced decrease in blood pressure, even when the heart rate is controlled by artificial cardiac stimulation. This depression of vasomotor activity includes inhibition of sympathetic drive, which can contribute to decreased cardiac contractility ${ }^{8}$. 
It has been supposed that neuralgic pain, only with extreme severity, may activate the vagoglossopharyngeal reflex, resulting in bradycardia, hypotension and syncope ${ }^{9}$, which could explain the disparity in the latency between the beginning of neuralgia and the syncope, observed in the majority of the cases reported.

The basic purpose of the treatment of glossopharyngeal neuralgia must be to control the pain, which may prevent bradycardia and syncope. This purpose can be reached through a variety of clinical, anesthetic and surgical techniques ${ }^{10}$. Local anesthetic blocking and spraying of the pharyngeal mucosa have been used for temporary relief. We have observed, in 1 patient, that this approach caused temporary relief of symptoms. Carbamazepine has proved useful in treating the idiopathic forms, and it can, sometimes, help to control the pain due to vascular intracranial compression $1,3,9$. The recommended dosage is 400 to $1,200 \mathrm{mg} /$ day. The problem with using carbamazepine is the possibility of tachyphylaxis due to the need of long-term use. Although about $20 \%$ of the patients who use carbamazepine can develop a benign leukopenia, they rarely have aplastic anemia ${ }^{11}$. One of the patients reported on here developed aplastic anemia associated with the use of carbamazepine.

Another interesting aspect of the use of carbamazepine is that its chronic use can, rarely, lead to severe sinus dysfunction and to atrioventricular conduction disturbances ${ }^{12}$

Phenytoin is seldom used to treat glossopharyngeal neuralgia due to its variable results ${ }^{1,3}$.

For the acute treatment of bradyarrhythmias, atropine and isoproterenol can be used with transitory results and no effect on the pain control ${ }^{3,9}$.

Artificial cardiac stimulation, as a way of preventing syncopal events, was originally proposed by Khero and Mullins ${ }^{7}$ in 1971 . Since then, provisory or permanent pacemakers have been used with variable results ${ }^{1,3,9}$.

The advantage of the use of a pacemaker is that, during the introduction of specific therapeutics, it can prevent bradyarrhythmias, during the paroxysmal episodes of pain. In both cases reported here, we observed remission of the syncopal events immediately after the beginning of stimulation through a temporary pacemaker, which allowed greater comfort during the period of adjustment and therapeutical definition. In our second case, the use of a temporary pacemaker was essential for the hemodynamic balance of the patient due to several episodes of asystole with low brain output and seizure.

The mechanism of cardiac syncope associated with glossopharyngeal neuralgia is a reflex and can be controlled after surgical or clinical treatment without being fatal. The majority of the authors do not recommend using a permanent pacemaker preventing the recurrence of syncopal events in these patients. Another important point is that artificial cardiac stimulation does not act on the primary cause of the syndrome, and it must be considered a palliative treatment. Jamshidi and Masrror ${ }^{5}$ treated their patients with carbamazepine and a permanent pacemaker, because they also had hypersensibility of the carotid sinus ${ }^{3}$. Ferrante et al ${ }^{3}$ recommended that intracranial surgery be considered, based on the evidence of the surgical series reported in of the literature and on the extreme disabling and distressing condition of the patient. The use of percutaneous radiofrequency lesions through the oval foramen has been proposed by some authors; however, it has been questioned because it can alter the vocal cord junction ${ }^{3}$. The surgical alternatives proposed include extracranial resection of the glossopharyngeal nerve by cervical and transtonsillar approaches, but they do not always yield favorable results ${ }^{3}$. Of the intracranial procedures, the first to offer convincing results was the original technique proposed by Dandy of resectioning of the glossopharyngeal nerve and of the upper vagal roots ${ }^{1,3}$. The latter is of value given the pathogenic mechanism of the syncope and also because it acts in the vagal nerve, which contributes to syncopal disorder.

Excellent results were obtained from microvascular decompression, which is, together with the Dandy procedure, the most appropriate and is relatively free of complications (excluding those that may arise from craniotomy at the posterior fossa level) ${ }^{3}$.

In conclusion, glossopharyngeal neuralgia associated with cardiac syncope is a clinical syndrome with a well-defined presentation, which, although usually limited and without reports of fatal cases, presents variable morbidity, demanding a prompt clinical diagnosis. The most appropriate initial approach, when no contraindication exists, is the introduction of a temporary pacemaker and therapeutic treatment with carbamazepine. An invasive approach can be necessary on an individual basis according to the etiological definition, refractoriness to medication, and clinical recurrence. 


\section{References}

1. Rushton JG, Stevens C, Miller RH. Glossopharyngeal (vagoglossopharyngeal) neuralgia; a study of 217 cases. Arch Neurol 1981; 98: 201-5.

2. Weinstein RE, Herec D, Friedman JH. Hypotension due to glossopharyngeal neuralgia. Arch Neurol 1986; 43: 90-2

3. Ferrante L, Artico M, Nardacci B, Fraioli B, Cosentino F, Fortuna A. Glossopharyngeal neuralgia with cardiac syncope. Neurosurgery 1995; 36: 58-63.

4. Chawla JC, Falconer MA. Glossopharyngeal and vagal neuralgia. Br Med J 1967; 3: 529- 31.

5. Jamshidi A, Masrror MA. Glossopharyngeal neuralgia with cardiac syncope. Arch Intern Med 1976; 136: 843-5.

6. Alpert JN, Armbrust Ca, Akhavi M, et al. Glossopharyngeal neuralgia, asystole, and seizures. Arch Neurol 1977; 34: 233-5.

7. Khero BA, Mullins CB. Cardiac syncope due to glossopharyngeal neuralgia. Arch Intern Med 1971; 128: 806-8.
8. Odeh M, Oliven A. Glossopharyngeal neuralgia associated with cardiac syncope and weight loss. Arch Otolaryng Head Neck Surg 1994; 120: 1283-6.

9. Johnston RT, Redding VJ. Glossopharyngeal neuralgia associated with cardiac syncope: long term treatment with permanent pacing and carbamazepine. $\mathrm{Br}$ Heart J 1990; 60: 403-5.

10. Ceylan S, Karakus A, Duru S, et al. Glossopharyngeal neuralgia: a study of 6 cases. Neurosurg Ver 1997; 20: 196-200.

11. Blackburn SC, Oliart AD, Garcia Rodriguez KA, Gutthanns P. Antiepileptics and blood dyscrasia: a cohort study. Pharmacotherapy 1998; 18: 1277-83.

12. Kasarskis EJ, Kuo CS, Berger R, Nelson KR. Carbamazepine-induced cardiac dysfunction: characterization of two distinct clinical syndromes. Arch Intern Med 1992; 152: 186-91.

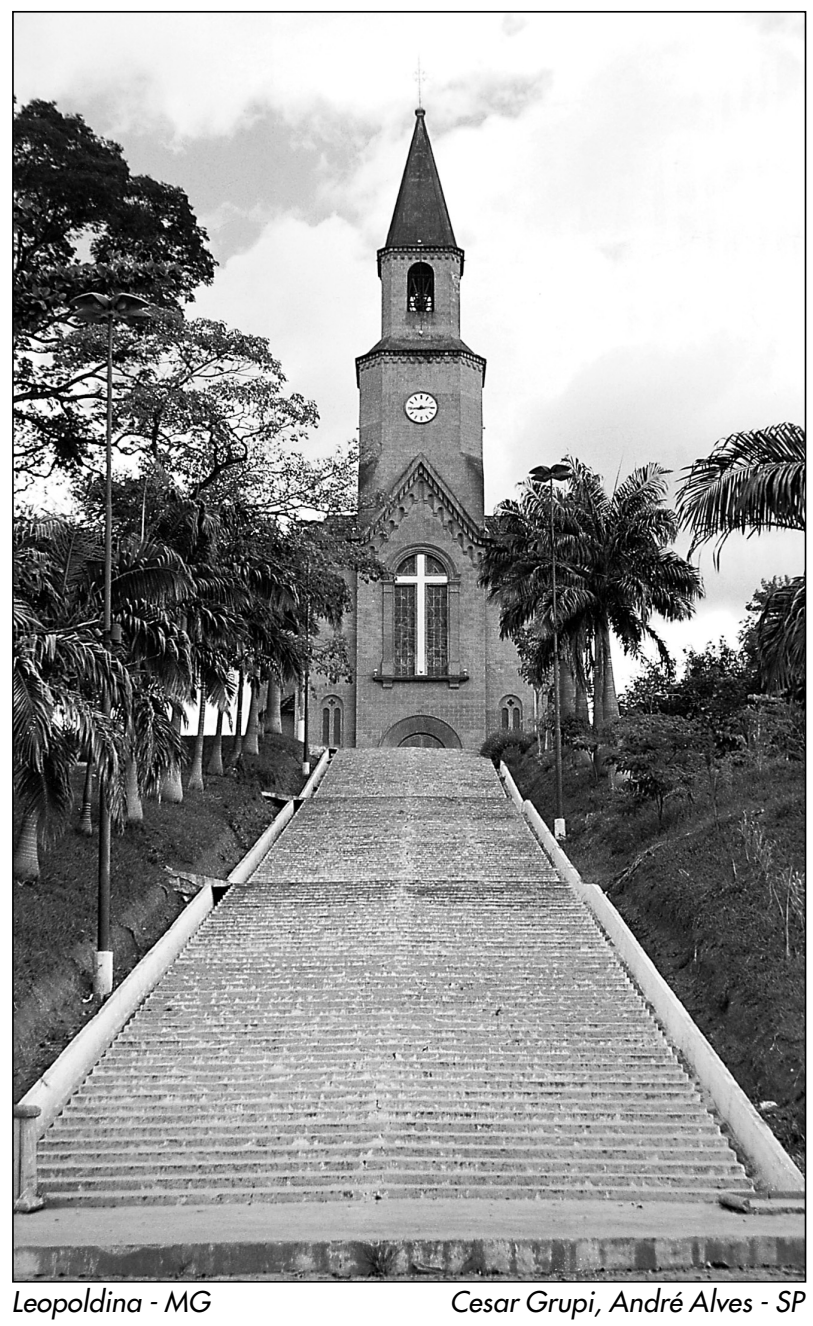

Editor da Seção de Fotografias Artísticas: Cícero Piva de Albuquerque

Correspondência: InCor - Av. Dr. Enéas C. Aguiar, 44 - 05403-000 - São Paulo, SP - E-mail: delcicero@incor. usp.br 